\title{
Stereotactic radiosurgery for single brain metastases from non-small cell lung cancer: progression of extracranial disease correlates with distant intracranial failure
}

\author{
Marie-Adele S Kress ${ }^{1 *}$, Eric Oermann², Matthew G Ewend ${ }^{2}$, Riane B Hoffman², Huma Chaudhry ${ }^{3}$ and Brian Collins ${ }^{1}$
}

\begin{abstract}
Background: Limited data exist regarding management of patients with a single brain lesion with extracranial disease due to non-small cell lung cancer (NSCLC).

Methods: Eighty-eight consecutive patients with a single brain lesion from NSCLC in the presence of extracranial disease were treated with stereotactic radiosurgery (SRS) alone. Local control (LC), distant intracranial failure (DIF), overall survival (OS), and toxicity were assessed. The logrank test was used to identify prognostic variables.

Results: Median OS was 10.6 months. One-year DIF was 61\%; LC 89\%. Treatments were delivered in 1-5 fractions to median $\mathrm{BED}_{10}=60 \mathrm{~Gy}$. Five patients developed radionecrosis. Factors associated with shortened OS included poor performance status (PS) $(p=0.0002)$ and higher Recursive Partitioning Analysis class $(p=0.017)$. For patients with PS 0 , median survival was 22 months. DIF was associated with systemic disease status (progressive vs. stable) ( $p=0.0001$ ), as was BED ( $p=0.021)$ on univariate analysis, but only systemic disease $(p=0.0008)$ on multivariate analysis.

Conclusions: This study identifies a patient population that may have durable intracranial control after treatment with SRS alone. These data support the need for prospective studies to optimize patient selection for up-front SRS and to characterize the impact of DIF on patients' quality of life.
\end{abstract}

Keywords: Brain metastases, Non-small cell lung cancer, Palliative care, Distant intracranial failure, Stereotactic radiosurgery, CyberKnife

\section{Background}

Brain metastases are common, occurring in $20-40 \%$ of cancer patients and contributing to $20 \%$ of annual cancer deaths [1]. Brain metastases are particularly common among patients with non-small cell lung cancer (NSCLC), even at the time of diagnosis, accounting for approximately $18-64 \%$ of all brain metastasis diagnoses [2]. However, some of these patients will present with limited intracranial disease, with one or few metastatic lesions. Overall prognosis of patients with brain metastases is limited, but has been shown to vary significantly, based on

\footnotetext{
* Correspondence: Marie-Adele.S.Kress@gunet.georgetown.edu 'Department of Radiation Medicine, Lombardi Comprehensive Cancer Center, Georgetown University Hospital, Lower Level Bles, 3800 Reservoir Road, N.W, Washington, DC 20007, USA

Full list of author information is available at the end of the article
}

factors such as tumor histology, number of lesions, patient age and performance status [3-7].

With advances in systemic therapy, tailoring intracranial radiation therapy (RT) to individual patients' clinical circumstances is increasingly important. Recent studies have evaluated the role of stereotactic radiosurgery (SRS) in combination with or instead of surgery and/or whole brain radiation therapy (WBRT) in carefully selected patient subgroups [8-10]. The goal of using SRS as a stand-alone therapy has been to maximize local control (LC) while minimizing toxicities and adverse impact on quality of life (QOL) [11-14]. However, some patients still benefit from up-front WBRT as part of their treatment, as demonstrated in Patchell's study where $24 \%$ of patients treated with surgery alone had distant intracranial recurrence only, compared to $8 \%$ who 
received adjuvant WBRT [14]. Guidelines exist, but remain vague, regarding optimal treatment regimens for patients with metastatic NSCLC; as a result, SRS use remains heterogeneous [15-19]. Overall, significant limitations still exist regarding optimizing patient selection for up-front SRS as a single modality, since these patients present with heterogeneous clinical circumstances.

Historically, a limited cohort of patients with a solitary brain lesion due to metastatic NSCLC have been treated with surgery to achieve LC and ultimately long-term intracranial disease-free survival $[20,21]$. However, in patients with single brain metastases in the presence of active extracranial disease, LC, distant intracranial failure (DIF) and survival are less while characterized. This patient population is important, since brain metastases are often diagnosed in the presence of extracranial disease. Both LC and DIF can be significant to patients' longterm prognosis as well as their functional status and QOL, making the decision of which up-front RT technique(s) to select particularly important.

To better define prognosis for patients with a single brain metastases from NSCLC and more effectively characterize which patients are at relatively high or low risk for distant intracranial recurrence, retrospective data from two institutions were pooled for evaluation of clinical outcomes and toxicity, as well as assessment of clinical prognostic variables.

\section{Methods}

\section{Patient selection}

This retrospective study was approved by the Institutional Review Boards of both Georgetown University and the University of North Carolina (UNC). Eightyeight patients were identified who were treated between 2002 and 2011, 40 of whom were treated at UNC, and 48 of whom were treated at Georgetown. All patients had a pathologically confirmed diagnosis of non-small cell lung cancer. Patients were included with any stage NSCLC at initial diagnosis, but at the time of presentation with brain metastases they had to have documented extracranial disease that was either stable or progressing. Patients were included if they had a single brain lesion, as confirmed by magnetic resonance imaging (MRI). Those with more than one intracranial lesion considered suspicious for metastasis, as documented by MRI, were excluded. Patients' performance statuses were estimated both using the Karnofsky Performance Status (KPS) and Eastern Cooperative Oncology Group (ECOG) scales, to allow for analysis by overall performance status (PS), Recursive Partitioning Analysis (RPA) classes and Diagnosis-Specific Graded Prognostic Index (DS-GPA) groups. Thirteen patients without radiographic followup were included in the analysis for overall survival (OS), but not for LC or DIF. One patient without any follow-up was not included in evaluation of OS, LC, or DIF.

\section{SBRT planning and treatment}

Each patient underwent simulation in the supine position with creation of a custom immobilization device. A treatment planning computed tomography (CT) scan with slices of 1-3 mm thickness was used for treatment of all patients. The majority of patients also had contrast-enhanced, thin-slice MRI fused with the CT scan for treatment planning; the most commonly used treatment planning sequence was magnetization-prepared rapid acquisition with gradient echo (MPRAGE), which is a high-resolution T1 sequence with contrast. Contrastenhanced CT scan was used at the discretion of the treating physician.

Gross tumor volume (GTV) was delineated on CT scan(s) and MRI (when applicable) after fusion in the MultiPlan treatment planning software. Typically, a circumferential margin of $1-3 \mathrm{~mm}$ was added to the GTV to create the clinical target volume (CTV), with no additional expansion to form the planning target volume (PTV). Adjacent critical structures were delineated as indicated, depending on the location of the treated tumor.

All treatments were performed using the CyberKnife system (Accuray, SunnyVale, CA) and were planned using Multiplan treatment software. This method uses an inverse-planning technique to generate conformal treatment plans with avoidance of critical structures. All treatments were delivered using $6 \mathrm{MV}$ photons and were prescribed to an isodose line that provided adequate ( $>95 \%)$ coverage of the PTV. All treatments were performed using Xsight cranial tracking. Biologic equivalent dose (BED) was calculated for each fractionation scheme, with $\alpha / \beta$ ratio assumed to be 10 , according to the following formula: $\mathrm{BED}_{10}=($ Prescription dose $) *(1+$ (Dose per fraction $/ \alpha / \beta)$ ).

\section{Other intracranial treatment}

Patients could not have undergone other intracranial therapy such as whole brain radiation therapy prior to this course of SRS. Patients were included regardless of receipt of previous systemic therapy or surgery. If patients were treated with surgery to an intracranial lesion, then the SRS treatment targeted the intracranial operative bed.

\section{Data collection and statistical analysis}

Patients were followed clinically by their treating radiation oncologist, neurosurgeon and/or medical oncologist after treatment. Radiographic follow-up was completed with contrast-enhanced MRI and was read by a specialist in neuroradiology. Typically, first radiographic follow-up occurred 1-2 months after treatment, with additional follow- 
up MRIs performed every 6 months, or sooner if patients presented with neurologic symptoms. Radiographic followup time was determined by the date on which progression was found or the last study that demonstrated no progression. Follow-up brain PET/CT imaging was used at the discretion of the treating physician, typically to differentiate between recurrence and radionecrosis.

Treatment response was evaluated according to the standard Response Evaluation Criteria in Solid Tumors (RECIST). LC was defined as no tumor growth after treatment. DIF was defined as the absence of any new intracranial lesions after treatment. LC, DIF, and OS were estimated from the date of the first fraction of SRS.

Actuarial OS, LC, and DIF were calculated using the Kaplan-Meier method. Patients were censored at the time of the measured event or at the time of last clinical followup. The social security death index was used to corroborate date of death. Univariate analyses were performed using the logrank test, and multivariate analyses were performed using Cox proportional-hazards regression.

Toxicities were evaluated according to the Common Terminology Criteria for Adverse Events (CTCAE), Version 4.0, with specific attention to rates of intracranial edema and radionecrosis. Determination of radionecrosis was made through collaboration of the treating radiation oncologist, neurosurgeon, neuroradiologist, and clinical findings, when appropriate.

\section{Results}

\section{Patient and lesion characteristics}

Detailed patient characteristics are presented in Table 1. A total of 88 patients were treated, 48 (55\%) at Georgetown, and 40 at UNC (45\%). The majority of the patients were male $(71 \%)$, with a median age of 65 . Patients generally had good performance status, with a median ECOG score of 1 (Table 1). All patients had systemic disease, equally divided among those with stable (43\%) or actively progressing (43\%) disease. Twelve patients had systemic disease, but its status as stable or progressive was unknown. The vast majority of patients (78\%) had additional sites of metastatic disease apart from the brain, and most patients were RPA class II, with only 4 patients in RPA class I, and 9 patients with unknown RPA status. Sixtyeight percent of patients were DS-GPA groups 1.5-2.5.

Variables relating to the intracranial lesions are presented in Table 2 . Notably, only $6 \%$ of patients underwent surgery prior to SRS, and $47 \%$ of patients had received at least one dose of chemotherapy prior to SRS treatment. Lesions overall were small, with a median size of $13 \mathrm{~mm}$ (range, 1.5-56 mm), as determined by MRI. Dosimetric parameters, presented in Table 3, demonstrated the various doses and fractionation schema used for treatment. Although all patients were treated using the CyberKnife system, some patients were treated in a single
Table 1 Patient characteristics

\begin{tabular}{ll}
\hline Characteristic & $\boldsymbol{n}(\%)$ \\
\hline Total patients & 88 \\
Site of treatment & \\
$\quad$ Georgetown University Hospital & $48(55 \%)$ \\
University of North Carolina & $40(45 \%)$ \\
Sex (n) (per patient) & \\
M & $71(81 \%)$ \\
F & $17(19 \%)$ \\
Age (y) (per patient) & \\
Median & 65 \\
Range & $37-84$ \\
ECOG Performance Status & \\
Median & 1 \\
0 & $26(30 \%)$ \\
1 & $30(34 \%)$ \\
2 & $18(20 \%)$ \\
3 & $4(5 \%)$ \\
4 & $2(2 \%)$ \\
Unknown & $8(9 \%)$
\end{tabular}

Status of systemic disease*

Stable $38(43 \%)$

Progressive $38(43 \%)$

Unknown $12(14 \%)$

Extracranial metastases**

$\begin{array}{ll}\text { Present } & 69(78 \%)\end{array}$

Absent $16(18 \%)$

Unknown 3 (3\%)

Recursive Partitioning Analysis Class

$\begin{array}{ll}1 & 4(5 \%) \\ 2 & 67(76 \%) \\ 3 & 8(9 \%) \\ \text { Unknown } & 9(10 \%)\end{array}$

* Systemic disease present, unknown to be stable vs. progressive, in 12 patients.

** Status unknown in 3 patients.

fraction, while others were treated in hypofractionated treatment plans of 2-5 fractions. The BED varied substantially, with a median $\mathrm{BED}_{10}$ of 60 (range, 28-81.6).

\section{Clinical outcomes}

Overall survival (OS), local control (LC) and distant intracranial failure (DIF) are summarized in Table 4. Of patients alive at last follow-up, median follow-up time was 11.1 months; median OS for the entire cohort was 10.6 months. One-year actuarial survival was $47.9 \%$. Patients had excellent 1-year actuarial LC of $89.1 \%$, and 1-year actuarial DIF of $61.2 \%$. 
Table 2 Lesion characteristics

\begin{tabular}{ll}
\hline Characteristic & $\boldsymbol{n}$ (\%) \\
\hline Location of lesion & 24 \\
Frontal & 19 \\
Parietal & 10 \\
Temporal & 11 \\
Occipital & 20 \\
Cerebellar & 4 \\
Other & $5(6 \%)$ \\
Surgery prior to SRS & $41(47 \%)$ \\
Chemotherapy prior to SRS & \\
Radiographic size (MRI, mm) & 13 \\
Median & $1.5-56$ \\
\hline
\end{tabular}

On univariate analysis (UVA), ECOG performance status $(\mathrm{p}=0.001)$ and RPA class $(\mathrm{p}=0.017)$ were associated with overall survival, but DS-GPA was not $(\mathrm{p}=0.32)$. When patients were placed into three groups: $\mathrm{ECOG}=0$; $\mathrm{ECOG}=1-2 ; \mathrm{ECOG}=3-4$, the association persisted $(\mathrm{p}=0.001$, Figure 1$)$. On multivariate analysis (MVA), only ECOG persisted as a significant factor $(\mathrm{p}=0.036$ when modeled with RPA class; $\mathrm{p}=0.0081$ when modeled without RPA class). No factors were found to be associated with LC on either UVA or MVA.

On UVA, both BED $(\mathrm{p}=0.021)$ and systemic disease status $(p=0.001$, Figure 2$)$ were found to be associated with DIF; only systemic disease status $(\mathrm{p}=0.001)$ persisted as a significant factor on MVA (Table 5). Using the Wilcoxon rank-sum test, systemic disease status

Table 3 Dosimetric parameters

\begin{tabular}{ll}
\hline Characteristic & $\boldsymbol{n}$ \\
\hline Prescribed dose, Gy & 22.6 \\
Mean & 20 \\
Median & $18-40$ \\
Range & \\
Number of fractions & 1 \\
Median & $1-5$ \\
Range & 2000 \\
Median dose per fraction & \\
BED ${ }_{10}$, Gy & 55.7 \\
Mean & 60 \\
Median & $28-81.6$ \\
Range & \\
Prescription isodose line, $\%$ & 81 \\
Mean & 80 \\
Median & $70-92$ \\
Range
\end{tabular}

Table 4 Clinical outcomes

\begin{tabular}{ll}
\hline Endpoint & Time (months) \\
\hline Median follow-up, living patients & 11.1 \\
Median survival & 10.6 \\
Median local control & 41.5 \\
Distant Intracranial Failure & \\
Median DIF, all patients & 17.3 \\
Median DIF, progressive disease & 6.2 \\
Median DIF, stable disease & Not reached \\
1-year outcomes & $\%$ Survival \\
1-year actuarial OS & $47.9 \%$ \\
1-year actuarial LC & $89.1 \%$ \\
1-year actuarial DIF & $61.2 \%$ \\
\hline
\end{tabular}

was not associated with radiographic follow-up time $(p=0.2701)$. A post-hoc power calculation to estimate the sensitivity to detect a difference in DIF based on systemic disease status was performed and predicted $99 \%$ power to detect type I/II error of 0.05 . A total of $11 \mathrm{pa}-$ tients (13\%) underwent a salvage course of RT, either to the previously treated lesion, a separate lesion within the brain, or a course of WBRT.

\section{Toxicity}

Toxicities were minimal. In the acute setting, four patients experienced radionecrosis, two had intracranial edema, two developed mild fatigue, and one developed mild headache. None of the acute toxicities required intervention. The four patients with acute radionecrosis were treated in a range of 1-3 fractions and had tumors ranging $13-23 \mathrm{~mm}$ in size. One additional patient developed late radionecrosis. Overall, a total of five patients developed radionecrosis at any time point (6\%). The majority of cases of radiation necrosis were asymptomatic and were found only on radiographic imaging.

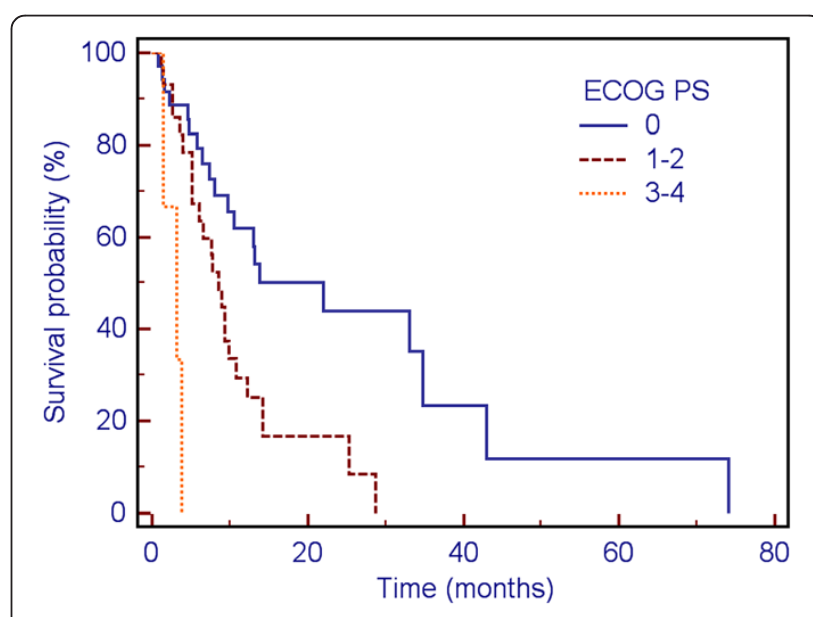

Figure 1 Impact of ECOG performance status on survival. 


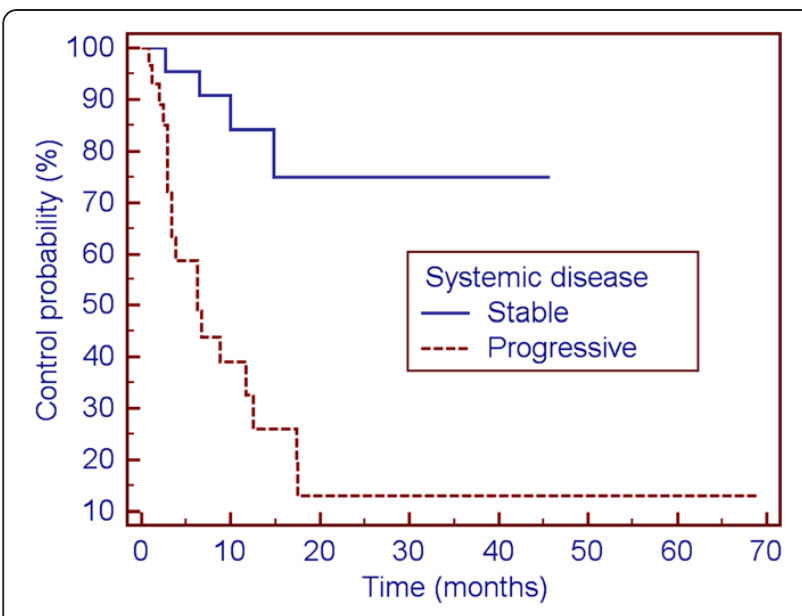

Figure 2 Impact of systemic disease status on distant intracranial failure.

Symptomatic radiation necrosis was treated with oral steroids. No patient required craniotomy for refractory radiation necrosis.

\section{Discussion}

Our study's novel finding is that the status of extracranial disease at the time of SRS treatment predicts more strongly than any other clinical or demographic variable for DIF. As a result, it may be possible to more effectively and prospectively identify patients who are most or least likely to have their intracranial disease entirely controlled by SRS alone. This criterion could significantly impact patient care by optimally selecting patients for SRS alone, while limiting the likelihood of needing salvage therapy.

Stereotactic radiosurgery (SRS) is increasingly being used for patients with brain metastases. Debate continues as to whether SRS is an appropriate first-line treatment without the additional use of surgery or whole brain radiation therapy (WBRT), and which endpoints, including OS, LC, DIF, and QOL are the most significant

Table 5 Multivariate analysis of distant intracranial failure

\begin{tabular}{llll}
\hline Variable & $\mathbf{p}$-value & $\operatorname{Exp}(\mathbf{b})$ & $\mathbf{9 5 \%} \mathbf{C l}$ of $\operatorname{Exp}(\mathbf{b})$ \\
\hline Systemic disease status & 0.0008 & 9.46 & $2.54-35.14$ \\
Treatment Site & 0.93 & 1.05 & $0.35-3.19$ \\
BED & 0.13 & 0.31 & $0.069-1.38$ \\
Size & 0.43 & 1.72 & $0.45-6.52$ \\
Surgery & 0.19 & 3.51 & $0.53-23.16$ \\
Age & 0.76 & 0.87 & $0.34-2.19$ \\
Extracranial metastases & 0.49 & 0.59 & $0.13-2.62$ \\
ECOG Performance Status & 0.59 & 1.18 & $0.64-2.18$ \\
Local Control & 0.61 & 0.54 & $0.05-5.69$ \\
\hline
\end{tabular}

in this patient population [16,22]. Limited data have demonstrated that in selected patients, an initial approach that includes SRS alone is appropriate $[11,16]$. However, these data are tempered by the fact that in clinical practice, many patients do not fit these trial criteria, including those of advanced age, with progressive extracranial disease, and/or poor performance status. As a result, many gaps remain in the literature regarding patient outcomes when using SRS alone as an initial approach. At the same time, the enthusiasm for embarking on SRS alone as initial therapy is necessarily tempered by the risks of development of distant intracranial disease, including risks of neurocognitive decline related to disease progression [14,23]. Although patient-reported outcomes (PROs)/QOL have not been studied among many subgroups of patients with brain metastases, it is also possible that an initial approach with SRS alone may lead to improved PROs/QOL for some who avoid or asymptomatically delay further intracranial treatment, while impaired long-term PROs/QOL for others who experience recurrence and require salvage therapy in the setting of pre-existing increase in symptoms. As a result, identification of factors predictive of distant intracranial recurrence is essential to refining patient selection for SRS, WBRT, or combination therapy.

Predicting survival of patients with brain metastases also has clinical utility. The first historical system developed for estimating patient prognosis, primarily during an era where treatment included WBRT and/or surgery, were the Recursive Partitioning Analysis (RPA) classes [5]. These classes, separating patients based upon age, status of primary tumor, and performance status, created rough prognostic groupings that, while useful, left large, heterogeneous groups of patients within the same category. For example, in our study, $76 \%$ of patients were considered RPA class II. A more modern system, the Diagnosis-Specific Graded Prognostic Index (DS-GPA), incorporates age, KPS, presence of extracranial metastases, and number of brain metastases to develop a score predictive of survival [6]. However, in our series, the DSGPA was not found to be significantly associated with survival, suggesting that there still exists significant heterogeneity, even among patients within similar prognostic groups. Even more interestingly, the majority (68\%) of the patients in this study had DS-GPA scores between $1.5-2.5$, which would result in a projected median survival of 6.53 months. Instead, we have demonstrated prolonged survival of over 10 months in this carefully selected patient group, which underscores the importance of considering patients' longer-term quality of life if they have limited brain metastases, even in the setting of additional adverse prognostic factors.

Although the present study has significant findings regarding survival and DIF, it does have several limitations 
due to its retrospective nature. Radiographic follow-up did not occur at consistent intervals for the entire study population, so events relating to LC and DIF may have been discovered, in some patients, with lag-time between development of disease and its recognition. The sample size of this study is still relatively small, and all power calculations were completed post-hoc due to the retrospective data collection and analysis. Additionally, no prospective quality of life measures or patient-reported outcomes were included in this study, which are of paramount importance in the palliative setting. Finally, patients were permitted to have additional systemic or local therapies at the discretion of their physicians, and preceding, concurrent, or adjuvant chemotherapy may have impacted DIF and OS in ways that were not analyzed in this study.

\section{Conclusions}

Patients with NSCLC with a single brain metastasis in the setting of residual extracranial disease live longer than might be predicted by traditional prognostic algorithms, including RPA class and DS-GPA. During their prolonged survival, both LC and DIF are remarkably high. The sole factor that increased the risk of distant intracranial failure was progressive systemic disease at the time of SRS. Prospective studies are needed to optimize patient selection for up-front SRS, and to refine follow-up schedules that minimize the impact of distant intracranial failure on patients' quality of life.

\section{Competing interest}

Brian Collins, MD is a paid speaker for Accuray. The remaining authors have no competing interests.

\section{Authors' contributions \\ This project was not supported by outside funding. MSK, EO, MGE, and BC all contributed to study concept and design. MSK, EO, and $\mathrm{HC}$ completed the data collection. MSK, EO, and RBH contributed to the data analysis. MSK contributed to drafting the manuscript. All authors contributing revising and giving final approval to the manuscript.}

\section{Disclosures}

Brian Collins, MD is a paid speaker for Accuray. The remaining authors have no financial disclosures.

\section{Meeting presentation}

This project was presented at ASTRO's 2012 annual meeting.

\section{Author details \\ ${ }^{1}$ Department of Radiation Medicine, Lombardi Comprehensive Cancer Center, Georgetown University Hospital, Lower Level Bles, 3800 Reservoir Road, N.W, Washington, DC 20007, USA. ${ }^{2}$ Department of Neurosurgery, University of North Carolina: Chapel Hill, 170 Manning Drive, CB 7060, Chapel Hill, NC 27599, USA. ${ }^{3}$ George Washington University, Ross Hall, 2300 Eye StreetNW, Washington DC 20037, USA.}

Received: 7 January 2013 Accepted: 7 March 2013

Published: 19 March 2013

\section{References}

1. Nussbaum ES, Djalilian HR, Cho KH, Hall WA: Brain metastases. Histology, multiplicity, surgery, and survival. Cancer 1996, 78:1781-1788.
2. Lassman AB, DeAngelis LM: Brain metastases. Neurol Clin 2003, 21(vii):1-23.

3. Borgelt B, Gelber R, Kramer S, Brady LW, Chang CH, Davis LW, Perez CA, Hendrickson FR: The palliation of brain metastases: final results of the first two studies by the Radiation Therapy Oncology Group. Int J Radiat Oncol Biol Phys 1980, 6:1-9.

4. Rades D, Kieckebusch S, Haatanen T, Lohynska R, Dunst J, Schild SE: Surgical resection followed by whole brain radiotherapy versus whole brain radiotherapy alone for single brain metastasis. Int J Radiat Oncol Biol Phys 2008, 70:1319-1324.

5. Gaspar L, Scott C, Rotman M, Asbell S, Phillips T, Wasserman T, McKenna WG, Byhardt R: Recursive partitioning analysis (RPA) of prognostic factors in three Radiation Therapy Oncology Group (RTOG) brain metastases trials. Int J Radiat Oncol Biol Phys 1997, 37:745-751.

6. Sperduto PW, Chao ST, Sneed PK, Suh JH, Luo X, Suh J, Roberge D, Bhatt A, Jensen AW, Brown PD, et al: Diagnosis-specific prognostic factors, indexes, and treatment outcomes for patients with newly diagnosed brain metastases: a multi-institutional analysis of 4,259 patients. Int J Radiat Oncol Biol Phys 2010, 77:655-661.

7. Patil CG, Pricola K, Sarmiento JM, Garg SK, Bryant A, Black KL: Whole brain radiation therapy (WBRT) alone versus WBRT and radiosurgery for the treatment of brain metastases. Cochrane Database Syst Rev 2012, 9: CD006121.

8. Sanghavi SN, Miranpuri SS, Chappell R, Buatti JM, Sneed PK, Suh JH, Regine WF, Weltman E, King VJ, Goetsch SJ, et al: Radiosurgery for patients with brain metastases: a multi-institutional analysis, stratified by the RTOG recursive partitioning analysis method. Int J Radiat Oncol Biol Phys 2001, 51:426-434.

9. Andrews DW, Scott CB, Sperduto PW, Flanders AE, Gaspar LE, Schell MC, Werner-Wasik M, Demas W, Ryu J, Bahary JP, et al: Whole brain radiation therapy with or without stereotactic radiosurgery boost for patients with one to three brain metastases: phase III results of the RTOG 9508 randomised trial. Lancet 2004, 363:1665-1675.

10. Aoyama H, Shirato H, Tago M, Nakagawa K, Toyoda T, Hatano K, Kenjyo M, Oya N, Hirota S, Shioura H, et al: Stereotactic radiosurgery plus wholebrain radiation therapy vs stereotactic radiosurgery alone for treatment of brain metastases: a randomized controlled trial. J Am Med Assoc 2006, 295:2486-2491.

11. Tsao M, Xu W, Sahgal A: A meta-analysis evaluating stereotactic radiosurgery, whole-brain radiotherapy, or both for patients presenting with a limited number of brain metastases. Cancer 2012, 118:2486-2493.

12. Chang EL, Wefel JS, Hess KR, Allen PK, Lang FF, Kornguth DG, Arbuckle RB, Swint JM, Shiu AS, Maor MH, Meyers CA: Neurocognition in patients with brain metastases treated with radiosurgery or radiosurgery plus wholebrain irradiation: A randomised controlled trial. Lancet Oncol 2009, 10:1037-1044.

13. Kondziolka D, Niranjan A, Flickinger JC, Lunsford LD: Radiosurgery with or without whole-brain radiotherapy for brain metastases: The patients' perspective regarding complications. Am J Clin Oncol 2005, 28:173-179.

14. Aoyama H, Tago M, Kato N, Toyoda T, Kenjyo M, Hirota S, Shioura H, Inomata T, Kunieda E, Hayakawa K, et al: Neurocognitive function of patients with brain metastasis who received either whole brain radiotherapy plus stereotactic radiosurgery or radiosurgery alone. Int J Radiat Oncol Biol Phys 2007, 68:1388-1395.

15. NCCN Clinical Practice Guidelines in Oncology (NCCN Guidelines): Central Nervous System Cancers Version 2. 2011. http://www.nccn.org/professionals/ physician_gls/pdf/cns.pdf.

16. Tsao MN, Rades D, Wirth A, Lo SS, Danielson BL, Gaspar LE, Sperduto PW, Vogelbaum MA, Radawski JD, Wang JZ, et al: Radiotherapeutic and surgical management for newly diagnosed brain metastasis(es): An American Society for Radiation Oncology evidence-based guideline. Practical Radiat Oncol 2012, 2:210-255.

17. Knisely JP, Yamamoto M, Gross CP, Castrucci WA, Jokura H, Chiang VL: Radiosurgery alone for 5 or more brain metastases: expert opinion survey. J Neurosurg 2010, 113:84-89.

18. Nieder C, Spanne O, Mehta MP, Grosu AL, Geinitz H: Presentation, patterns of care, and survival in patients with brain metastases: what has changed in the last 20 years? Cancer 2010, 117:2505-2512.

19. Halasz LM, Weeks JC, Neville BA, Taback N, Punglia RS: Use of Stereotactic Radiosurgery For Brain Metastases: A SEER-Medicare Analysis. Int J Radiat Oncol Biol Phys 2011, 81(2):S550-S551. 
20. Patchell RA, Tibbs PA, Walsh JW, Dempsey RJ, Maruyama Y, Kryscio RJ, Markesbery WR, Macdonald JS, Young B: A randomized trial of surgery in the treatment of single metastases to the brain. N Engl J Med 1990, 322:494-500.

21. Patchell RA, Tibbs PA, Regine WF, Dempsey RJ, Mohiuddin M, Kryscio RJ, Markesbery WR, Foon KA, Young B: Postoperative radiotherapy in the treatment of single metastases to the brain: a randomized trial. J Am Med Assoc 1998, 280:1485-1489.

22. Scoccianti S, Ricardi U: Treatment of brain metastases: review of phase III randomized controlled trials. Radiother Oncol 2012, 102:168-179.

23. Regine WF, Scott C, Murray K, Curran W: Neurocognitive outcome in brain metastases patients treated with accelerated-fractionation vs. accelerated-hyperfractionated radiotherapy: An analysis from Radiation Therapy Oncology Group Study 91-04. Int J Radiat Oncol Biol Phys 2001, 51:711-717.

doi:10.1186/1748-717X-8-64

Cite this article as: Kress et al: Stereotactic radiosurgery for single brain metastases from non-small cell lung cancer: progression of extracranial disease correlates with distant intracranial failure. Radiation Oncology $20138: 64$

\section{Submit your next manuscript to BioMed Central and take full advantage of:}

- Convenient online submission

- Thorough peer review

- No space constraints or color figure charges

- Immediate publication on acceptance

- Inclusion in PubMed, CAS, Scopus and Google Scholar

- Research which is freely available for redistribution 